\title{
Finite-Difference Numerical Methods for Solving the Energy-Momentum Transport Equations in Two-Valley Semiconductors*
}

\author{
R. K. Mains, M. A. El-Gabaly, and G. I. Haddad \\ Solid-State Electronics Laboratory, \\ Department of Electrical and Computer Engineering, \\ University of Michigan, Ann Arbor, Michigan 48109-1109 \\ Received June 5, 1984; revised August 30, 1984
}

\begin{abstract}
Two finite-difference methods for solving the energy-momentum transport equations for electrons in two-valley semiconductors are analyzed. For each method, stability analyses are carried out including the electric field terms and relaxation terms in the equations. Results of large-signal simulations of GaAs IMPATTs using these numerical methods are presented and compared. 1985 Academic Press, Inc.
\end{abstract}

\section{INTRODUCTION}

The energy-momentum transport model is useful for simulating carrier dynamics in III-V compound semiconductors at frequencies where the quasi-static, drift-diffusion transport model is no longer adequate [1-4]. However, significant numerical problems are introduced by including velocity and energy transport equations. These equations allow wave propagation at speeds close to the average thermal velocity of carriers [5], typically at least an order of magnitude higher than the average carrier velocity. By the Courant-Friedrich-Lewy stability restriction [6], this implies that the time step for an energy-momentum simulation is at least ten times smaller than the time step for a comparable drift-diffusion simulation. Therefore to minimize computer time, it is desirable to use simple numerical methods to solve these equations.

In addition, some of the finite-difference numerical techniques that work well for drift-diffusion simulations, such as one-step explicit forward time-upwind drift differencing [7], can cause numerical instability problems when applied to the velocity and energy transport equations.

This paper considers two finite-difference algorithms for solving the energymomentum transport equations for electrons in two-valley semiconductors. Both are one-step methods in the sense that the equations are solved just once with each

\footnotetext{
* This work was supported by the Air Force Systems Command, Avionics Laboratory, Wright-Patterson Air Force Base, Ohio Contract No. F33615-81-K-1429.
} 
time step advancement. Two-step methods, such as the Lax-Wendroff type $[6,8]$, are not considered partially due to the additional calculations necessary to implement these methods and partially because they have not been as successful as the one-step methods in producing smooth, stable solutions during the course of this work.

\section{Transport Equations for Two-Valley Semiconductors}

The following normalized quantities are introduced:

$$
\begin{array}{llrl}
\underline{n}_{i}=n_{i}\left(\frac{q^{2} \Delta t^{2}}{\varepsilon}\right), & \underline{v}_{i}=v_{i}\left(\frac{\Delta t}{\Delta x}\right), & \underline{w}_{i}=w_{i}\left(\frac{\Delta t^{2}}{m_{i}^{*} \Delta x^{2}}\right), \\
\underline{E}=E\left(\frac{q \Delta t^{2}}{\Delta x}\right), & \underline{\tau}=\frac{\tau}{\Delta t}, & \underline{\tau}_{(i-j)}=\tau_{(i-j)} \frac{m_{j}^{*}}{\Delta t m_{i}^{*}},
\end{array}
$$

where the lower bar denotes a normalized quantity, $n_{i}$ is the average particle concentration in valley $i$ in $\mathrm{m}^{-3}, v_{i}$ is the average per-carrier velocity in $\mathrm{m} / \mathrm{s}, w_{i}$ is the average per-carrier energy in joules, $E$ is the electric field in $\mathrm{V} / \mathrm{m}$, and $\tau, \tau_{(i-j)}$ denote relaxation times in seconds. The (constant) time and space steps used in the simulation are $\Delta t$ and $\Delta x$ in seconds, $m, q$ (positive) is the electronic charge in coulombs, $\varepsilon$ is the permittivity in $\mathbf{F} / \mathrm{m}$, and $m_{i}^{*}$ is the effective mass in kilograms in valley $i$. The indices $(i, j)$ can take on the values $(1,2)$ or $(2,1)$ and $\tau_{(i-j)}$ characterizes a process involving nonequivalent intervalley transitions from valley $i$ to valley $j$. The purpose of the normalizations in (1) is to minimize the necessary multiplications and divisions by constant factors in the transport equations. This is not, of course, the only set of normalizations that could be used. For example, $n_{i}$ and $E$ could be further normalized by dividing by either $m_{1}^{*}$ or $m_{2}^{*}$, in which case the ratio of effective masses would appear in the final form of the normalized transport equations.

Adopting the normalizations in (1) yields the 1-dimensional transport equations:

$$
\begin{aligned}
& \delta_{i} \underline{n}_{i}=-\delta_{x}\left(\underline{n}_{i} \underline{v}_{i}\right)-\frac{\underline{n}_{i}}{\underline{\tau}_{m i}\left(w_{i}\right)}+\frac{\underline{n}_{j}}{\tau_{n j}\left(w_{j}\right)}, \\
& \delta_{i} \underline{v}_{i}=-\frac{1}{2} \delta_{x} \underline{v}_{i}^{2}-\frac{\underline{E}}{m_{i}^{*}}-\frac{2}{3 \underline{n}_{i}} \delta_{x}\left[\underline{n}_{i}\left(\underline{w}_{i}-\frac{1}{2} \underline{v}_{i}^{2}\right)\right]-\frac{\underline{v}_{i}}{\underline{\tau}_{m i}\left(w_{i}\right)}
\end{aligned}
$$

and

$$
\begin{aligned}
\delta_{t} \underline{w}_{i}= & -\underline{\underline{v}}_{i} \delta_{x} \underline{w}_{i}-\frac{\underline{E}}{m_{i}^{*}} \underline{\underline{w}}_{i}-\frac{2}{3 \underline{n}_{i}} \delta_{x}\left[\underline{\underline{n}}_{i} \underline{\underline{w}}_{i}\left(\underline{w}_{i}-\frac{1}{2} \underline{v}_{i}^{2}\right)\right]-\frac{\underline{w}_{i}-\underline{w}_{i \mathrm{th}}}{\underline{\tau}_{e i}\left(w_{i}\right)} \\
& -\frac{\underline{w}_{i}}{\underline{\tau}_{e(i-j)}\left(w_{i}\right)}-\frac{\underline{w}_{j}}{\underline{\underline{\tau}} \underline{\underline{e}}_{(j-i)}\left(w_{j}\right)} \frac{\underline{n}_{j}}{\underline{n}_{i}} .
\end{aligned}
$$


The relaxation times in (2) through (4) are explained in [1]. The quantity $\underline{w}_{i t h}$ is the lattice thermal energy (3/2) $k T_{0}$, where $k$ is Boltzmann's constant and $T_{0}$ is the lattice temperature normalized by $\Delta t^{2} / m_{i}^{*} \Delta x^{2}$. Note that ionization is not included in (2)-(4) since the stability of the numerical methods will be examined in the lowenergy limit. In addition to (2)-(4), Poisson's equation is used to solve for the electric field $E . \delta_{t}$ and $\delta_{x}$ denote any finite difference in time and space, respectively; the specific form of these operators determines the numerical method used.

\section{ForWARD TIME-UPWIND DRIFT DIFFERENCING}

Using forward-time differencing for $\delta_{1}$ and upwind-drift differencing for $\delta_{x}$ when applied to the transportive terms yields:

$$
\begin{aligned}
n_{i}^{k(t+\Delta t)}-n_{i}^{k(t)}= & -\left[n_{i}^{k(t)} v_{i}^{k(t)}-n_{i}^{(k-1)(t)} v_{i}^{(k-1)(t)}\right] \\
& -\frac{n_{i}^{k(t+\Delta t)}}{\tau_{n i}^{k}\left[w_{i}^{k(t)}\right]}+\frac{n_{j}^{k(t+\Delta t)}}{\tau_{n j}^{k}\left[w_{j}^{k(t)}\right]}, \\
v_{i}^{k(t+\Delta t)}-v_{i}^{k(t)}= & -\frac{1}{2}\left[\left(v_{i}^{k(t)}\right)^{2}-\left(v_{i}^{(k-1)(t)}\right)^{2}\right]-\frac{E^{k(t)}}{m_{i}^{*}} \\
& -\frac{\left[\begin{array}{l}
n_{i}^{(k+1)(t)}\left[w_{i}^{(k+1)(t)}-\frac{1}{2}\left(v_{i}^{(k+1)(t)}\right)^{2}\right] \\
\left.-n_{i}^{(k-1)(t)}\left[w_{i}^{(k-1)(t)}-\frac{1}{2}\left(v_{i}^{(k-1)(t)}\right)^{2}\right]\right] \\
3 n_{i}^{k(t)}
\end{array}\right.}{} \\
& -\frac{v_{i}^{k(t+\Delta t)}}{\tau_{m i}^{k}\left(w_{i}^{k(t)}\right)},
\end{aligned}
$$

and

$$
\begin{aligned}
& w_{i}^{k(t+\Delta t)}-w_{i}^{k(t)} \\
& =-v_{i}^{k(t)}\left(w_{i}^{k(t)}-w_{i}^{(k-1)(t)}\right)-E^{k(t)} \frac{v_{i}^{k(t)}}{m_{i}^{*}} \\
& -\frac{2}{3 n_{i}^{k(t)}}\left\{n_{i}^{k(t)} v_{i}^{k(t)}\left[w_{i}^{k(t)}-\frac{1}{2}\left(v_{i}^{k(t)}\right)^{2}\right]-n_{i}^{(k-1)(t)} v_{i}^{(k-1)(t)}\right. \\
& \left.\times\left[w_{i}^{(k-1)(t)}-\frac{1}{2}\left(v_{i}^{(k-1)(t)}\right)^{2}\right]\right\}-\frac{w_{i}^{k(t+\Delta t)}-w_{i \text { th }}}{I_{e i}^{k}\left(w_{i}^{k(t)}\right)}-\frac{w_{i}^{k(t+\Delta t)}}{\tau_{e(i-j)}^{k}\left(w_{i}^{k(t)}\right)} \\
& +\frac{w_{j}^{k(t+\Delta t)}}{\underline{\underline{\tau_{e(j-i)}^{k}}\left(w_{j}^{k(t)}\right)}} \frac{n_{j}^{k(t+\Delta t)}}{n_{i}^{k(t+\Delta t)}} \text {. }
\end{aligned}
$$

In (5)-(7) the subscripts $i, j$ again denote valley indices; the first superscripts denote adjacent meshpoints at $(k-1), k$, and $(k+1)$ and the second superscripts 
denote present time $(t)$ or future time $(t+\Delta t)$, where quantities at the present time are known and quantities at $(t+\Delta t)$ are to be obtained by solving (5)-(7) once. The lower bars are omitted for the $n, v, E$, and $w$ terms in (5)-(7), however, it is assumed that quantities specified at meshpoints are normalized according to (1). It was assumed in (5)-(7) that the electric field $E^{k}$ points to the left and is negative and that the particle velocities $v_{i}^{k}$ are to the right and positive. The mesh is set up so that point indices increase to the right; therefore, the meshpoints $k$ and $(k-1)$ are used to form the upwind derivative in the transportive terms. The pressure gradient term in the velocity equation (6) uses space-centered differencing for $\delta_{x}$. Of course the simulation must check for negative velocities and points of velocity reversals at each iteration and the form of the upwind derivative must be modified according to the discussions in [6] under these circumstances.

It should be noted that one advantage of this numerical method is that (5) is conservative so that particles are not artificially created or destroyed by the algorithm. Also, it is noted that since the relaxation terms in (5)-(7) are evaluated at future time, these equations for the two valleys are coupled and must be solved simultaneously [1].

The stability analysis is carried out by first assuming that a uniform, dc solution of (5)-(7) has been obtained. It is then assumed that a small error is linearly superimposed on the dc solution, which for $n_{i}^{k(t)}$ is expressed as:

$$
\tilde{n}_{i}^{k(t)}=\sum_{l} \tilde{\hat{n}}_{i i}^{(t)} e(j k l \Delta x)
$$

with similar expressions for the errors $\tilde{v}_{l}^{k(t)}$ and $\tilde{w}_{l}^{k(t)}$. The terms $\tilde{n}_{l}, \tilde{\hat{v}}_{l}$, and $\tilde{w}_{l}$ are the amplitudes of the $l$ th Fourier mode in the error decomposition, where $l=m \pi / M \Delta x$, $M$ is the number of meshpoints, and $m$ is an integer such that $|m| \leqslant M$, and $j$ in (8) is $\sqrt{-1}$. The de solution plus error terms is substituted in (5)-(7), the de terms are canceled out, and an equation to first order in the error terms is obtained for the change of the Fourier mode amplitudes across one time step which has the form

$$
\left[\begin{array}{c}
\tilde{n}_{1 l} \\
\tilde{\hat{v}}_{1 l} \\
\tilde{\hat{w}}_{1 l} \\
\tilde{\hat{n}}_{2 l} \\
\tilde{\hat{v}}_{2 l} \\
\hat{w}_{2 l}
\end{array}\right]_{(t+\Delta t)}=[G]\left[\begin{array}{c}
\tilde{n}_{1 l} \\
\hat{\hat{v}}_{1 l} \\
\hat{w}_{1 l} \\
\tilde{\hat{n}}_{2 l} \\
\hat{v}_{2 l} \\
\tilde{\hat{w}}_{2 l}
\end{array}\right]_{(t)}
$$

$G$ in $(9)$ is a $6 \times 6$ matrix, called the amplification matrix. For stability, it is required that all eigenvalues $\lambda$ of the matrix $G$ be such that $|\lambda| \leqslant 1$ [9]. To specify the $G$ matrix entries, use is made of the following definitions: 


$$
\begin{aligned}
& T_{1}=1+\frac{1}{\underline{\tau}_{n 1}}+\frac{1}{\underline{\tau}_{n 2}}, \quad T_{2}=1+\frac{1}{\underline{\tau}_{n 2}}, \quad F_{1}=\left(1-e^{-j l \Delta x}\right), \\
& W_{i}=\left(\underline{w}_{i}-\frac{1}{2} \underline{v}_{i}^{2}\right), \quad T_{3}=1+\frac{1}{\underline{\tau}_{m 1}}, \quad S=\sin (l \Delta x), \\
& C=\cos (l \Delta x), \\
& T_{4}=\left(1+\frac{1}{\underline{\tau}_{e 2}}+\frac{1}{\underline{\tau}_{e(2-1)}}\right), \\
& T_{5}=\left(1+\frac{1}{\underline{\tau}_{e 1}}+\frac{1}{\underline{\tau}_{e(1-2)}}\right), \quad T_{6}=T_{4} \times T_{5}-\frac{1}{\underline{\tau}_{e(1-2)} \underline{\underline{\tau} e(2-1)}}, \\
& T_{7}\left(1+\frac{1}{\tau_{n 1}}\right), \quad T_{8}=1+\frac{1}{\underline{\tau}_{m 2}} .
\end{aligned}
$$

Then the $G$ matrix for forward time-upwind drift differencing is:

$$
\begin{aligned}
& g_{11}=\frac{T_{2}\left(1-\underline{v}_{1} F_{1}\right)}{T_{1}}, \quad g_{12}=-\frac{T_{2} F_{1}}{T_{1}}, \\
& g_{14}=\frac{\frac{1}{\tau_{n 2}}\left(1-\underline{v}_{2} F_{1}\right)}{T_{1}} \\
& \begin{array}{l}
g_{15}=-\frac{F_{1}}{I_{n 2} T_{1}} \\
g_{23}=-\frac{2 j S}{3 T_{3}},
\end{array} \\
& g_{21}=-\frac{2 j W_{1} S}{3 T_{3}} \\
& g_{22}=\frac{1-\underline{v}_{1} F_{1}+\frac{2 j}{3} \underline{v}_{1} S}{T_{3}} \\
& g_{33}=-\frac{T_{4}\left[1-\frac{5}{3} \underline{v}_{1} F_{1}\right]}{T_{6}}, \quad g_{34}=-\frac{2 \underline{v}_{2} W_{2} F_{1}}{3 \underline{\underline{\tau}} e(2-1) T_{6}}, \\
& g_{35}=-\frac{\frac{2}{3} W_{2} F_{1}+\frac{E}{m_{2}^{*}}}{\underline{\underline{\tau}}_{e(2-1)} T_{6}}, \quad g_{36}=\frac{1-\frac{5}{3} \underline{v}_{2} F_{1}}{\underline{\underline{\tau}}_{e(2-1)} T_{6}}, \quad g_{41}=\frac{1-\underline{v}_{1} F_{1}}{\underline{\tau}_{n 1} T_{1}}, \\
& g_{42}=-\frac{F_{1}}{\underline{\tau}_{n 1} T_{1}}, \\
& g_{44}=\frac{T_{7}\left[1-\underline{v}_{2} F_{1}\right]}{T_{1}}, \quad g_{45}=-\frac{T_{7} F_{1}}{T_{1}}, \\
& g_{54}=-\frac{2 j W_{2} S}{3 T_{8}} \\
& g_{55}=\frac{1-\underline{v}_{2} F_{1}+\frac{2 j}{3} \underline{v}_{2} S}{T_{8}}, \quad g_{56}=-\frac{j 2 S}{3 T_{8}},
\end{aligned}
$$




$$
\begin{array}{ll}
g_{61}=-\frac{2 \underline{v}_{1} W_{1} F_{1}}{3 \underline{\underline{\tau}} e(1-2) T_{6}}, & g_{62}=-\frac{\frac{2}{3} W_{1} F_{1}+\frac{\underline{E}}{m_{1}^{*}}}{\underline{\underline{\tau} e(1-2)} T_{6}}, \\
g_{63}=\frac{1-\frac{5}{3} \underline{v}_{1} F_{1}}{\underline{\underline{\tau}} e(1-2) T_{6}}, & g_{64}=-\frac{2 \underline{v}_{2} T_{5} W_{2} F_{1}}{3 T_{6}}, \\
g_{65}=\frac{T_{5}\left[-\frac{2}{3} W_{2} F_{1}-\frac{E}{m_{2}^{*}}\right]}{T_{6}}, & g_{66}=\frac{T_{5}\left[1-\frac{5}{3} \underline{v}_{2} F_{1}\right]}{T_{6}} .
\end{array}
$$

Remaining entries not specified in (11) are zero.

\section{Modified LaX Numerical Method}

Use of the Lax finite-difference algorithm increases the stability of the energymomentum transport equations, however, at the price of increased numerical diffusion in the solution [6]. In practice it has been found that the numerical diffusion can be reduced to acceptable limits by proper choice of $\Delta x$ and $\Delta t$. Another drawback of the Lax method is that the transportive terms in the equations are no longer conservative so that carrier multiplication is not strictly related to the ionization rate in IMPATT simulation. To overcome this difficulty, a modified Lax method was used wherein the particle equation is solved using the previous forward time-upwind drift differencing, while the velocity and energy equations are solved using Lax's method. Therefore the finite-difference equation for particles is identical to (5) and the remaining equations become:

$$
\begin{aligned}
v_{i}^{k(t+\Delta t)}- & \frac{1}{2}\left[v_{i}^{(k-1)(t)}+v_{i}^{(k+1)(t)}\right] \\
= & -\frac{1}{4}\left[\left(v_{i}^{(k+1)(t)}\right)^{2}-\left(v_{i}^{(k-1)(t)}\right)^{2}\right]-\frac{E^{k(t)}}{m_{i}^{*}} \\
& -\frac{\left\{\begin{array}{c}
n_{i}^{(k+1)(t)}\left[w_{i}^{(k+1)(t)}-\frac{1}{2}\left(v_{i}^{(k+1)(t)}\right)^{2}\right] \\
\left.-n_{i}^{(k-1)(t)}\left[w_{i}^{(k-1)(t)}-\frac{1}{2}\left(v_{i}^{(k-1)(t)}\right)^{2}\right]\right\}
\end{array}-\frac{v_{i}^{k(t+\Delta t)}}{\mathbb{I}_{m i}^{k}\left(w_{i}^{k(t)}\right)}\right.}{(3)\left(\frac{1}{2}\right)\left[n_{i}^{(k-1)(t)}+n_{i}^{(k+1)(t)}\right]}
\end{aligned}
$$

and

$$
\begin{aligned}
w_{i}^{k(t+} & \Delta t)-\frac{1}{2}\left[w_{i}^{(k-1)(t)}+w_{i}^{(k+1)(t)}\right] \\
& =-\frac{1}{4}\left[v_{i}^{(k-1)(t)}+v_{i}^{(k+1)(t)}\right] \cdot\left[w_{i}^{(k+1)(t)}-w_{i}^{(k-1)(t)}\right]-\frac{E^{k(t)}}{2 m_{i}^{*}}\left[v_{i}^{(k-1)(t)}+v_{i}^{(k+1)(t)}\right]
\end{aligned}
$$




$$
\begin{aligned}
& -\left\{\begin{array}{c}
n_{i}^{(k+1)(t)} v_{i}^{(k+1)(t)}\left[w_{i}^{(k+1)(t)}-\frac{1}{2}\left(v_{i}^{(k+1)(t)}\right)^{2}\right] \\
-n_{i}^{(k-1)(t)} v_{i}^{(k-1)(t)}\left[w_{i}^{(k-1)(t)}-\frac{1}{2}\left(v_{i}^{(k-1)(t)}\right)^{2}\right]
\end{array}\right\} \\
& -\frac{\left.w_{i}\right)\left[n_{i}^{(k-1)(t)}+n_{i}^{(k+1)(t)}\right]}{w_{i}^{k(t+\Delta t)}-\underline{w}_{i t \mathrm{t}}}-\frac{w_{i}^{k(t+\Delta t)}}{\underline{\tau}_{e i}^{k}\left(w_{i}^{k(t)}\right)}+\frac{w_{j}^{k(t+\Delta t)}}{\underline{\tau}_{e(i-j)}^{k}\left(w_{i}^{k(t)}\right)}+\frac{n_{j}^{k(t+\Delta t)}}{\underline{\tau}_{e(j-i)}^{k}\left(w_{j}^{k(t)}\right)} \frac{n_{i}^{k(t+\Delta t)}}{}
\end{aligned}
$$

In (12) and (13), space-averaged quantities appear in the right-hand side in addition to the space-averaged quantity on the left-hand side called for by the Lax $\delta_{t}$ operator. This choice of additional averaged quantities was found necessary to prevent numerically induced "wiggles" [6] from appearing in the solutions. All spatial derivatives in (12) and (13) are space centered as prescribed by the Lax method [6]. The amplification matrix entries for this modified Lax numerical method are:

$$
\begin{aligned}
& g_{22}=\frac{C-\frac{j}{3} \underline{v}_{l} S}{T_{3}}, \quad g_{31}=-\frac{2 j T_{4} S \underline{v}_{1} W_{1}}{3 T_{6}}, \\
& g_{32}=\frac{T_{4}\left(\frac{\underline{E}}{m_{1}^{*}} C-\frac{2 j}{3} S W_{1}\right)}{T_{6}}, \quad g_{33}=\frac{T_{4}\left(C-\frac{5 j}{3} \underline{v}_{1} S\right)}{T_{6}}, \\
& g_{34}=-\frac{2 j S \underline{v}_{2} W_{2}}{3 \underline{\underline{\tau}}_{e(2-1)} T_{6}}, \quad g_{35}=\frac{\frac{\underline{E}}{m_{2}^{*}} C-\frac{2 j}{3} S W_{2}}{\underline{\underline{\tau}}_{e(2-1)} T_{6}}, \\
& g_{36}=\frac{C-\frac{5 j}{3} \underline{v}_{2} S}{\underline{\underline{\tau} e(2-1)} T_{6}}, \quad g_{55}=\frac{C-j \frac{\underline{v}_{2}}{3} S}{T_{8}}, \quad g_{61}=-\frac{2 j S \underline{\underline{v}}_{1} W_{1}}{3 \underline{\underline{\tau}} e(1-2)_{6} T_{6}} \\
& g_{62}=\frac{\frac{\underline{E}}{m_{1}^{*}} C-\frac{2 j}{3} S W_{1}}{\underline{\underline{\tau}} e(1-2) T_{6}}, \quad g_{63}=\frac{C-j \frac{5}{3} \underline{\nu}_{1} S}{\underline{\underline{\tau}} e(1-2) T_{6}}, \quad g_{64}=-\frac{2 j S T_{5} \underline{\nu}_{2} W_{2}}{3 T_{6}}, \\
& g_{65}=\frac{T_{5}\left(\frac{E}{m_{2}^{*}} C-\frac{2 j}{3} S W_{2}\right)}{T_{6}}, \quad g_{66}=\frac{T_{5}\left(C-j \frac{5}{3} \underline{v}_{2} S\right)}{T_{6}} .
\end{aligned}
$$

Matrix entries not specified in (14) are the same as for the previous method given in [11]. 
TABLE I

Parameters Used to Calculate Amplification Matrix Entries in the Low Electric Field, Low Energy Limit

$$
\begin{aligned}
\tau_{n 1} & =9.687 \times 10^{-12} \mathrm{~s} \\
\tau_{n 2} & =5.958 \times 10^{-13} \mathrm{~s} \\
v_{1} & =v_{2}=E=0 \\
\tau_{e 2} & =1.696 \times 10^{-13} \mathrm{~s} \\
\tau_{e(2-1)} & =5.259 \times 10^{-13} \mathrm{~s} \\
\tau_{e(2-1)}^{*} & =9.114 \times 10^{-14} \mathrm{~s}
\end{aligned}
$$

$$
\begin{aligned}
\tau_{m 1} & =1.573 \times 10^{-13} \mathrm{~s} \\
\tau_{m 2} & =3.504 \times 10^{-14} \mathrm{~s} \\
\tau_{e 1} & =4.066 \times 10^{-13} \mathrm{~s} \\
\tau_{e(1-2)} & =1.549 \times 10^{-12} \mathrm{~s} \\
\tau_{e(1-2)}^{*} & =8.925 \times 10^{-12} \mathrm{~s} \\
w_{1} & =w_{2}=0.0646 \mathrm{eV}
\end{aligned}
$$

Note. Asterisk denotes relaxation times with double bars in the energy equation.

\section{Results of Stability ANALYsis AND COMPARISON OF METHODS}

The stability analysis was carried out as follows: A particular mesh space step was first selected (in these results, $D X=0.01 \mu \mathrm{m}$ and $0.02 \mu \mathrm{m}$ were both examined). Relaxation times, dc velocities, and energies corresponding to particular electric field values had been determined previously by Monte Carlo simulations for GaAs at $T=500^{\circ} \mathrm{K}[1]$; these values were used to calculate the amplification matrix entries for different values of time step $\Delta t$. (This temperature was used for the Monte Carlo calculations because it is a typical operating temperature for IMPATT diodes in the CW mode or in pulsed mode with high duty cycle). For each $\Delta t$, the eigenvalues of the amplification matrix were scanned over the entire range of $l$ the Fourier mode index. If the magnitudes of these eigenvalues were all less than or equal to one, the method was considered stable for that particular $\Delta t$. If at least one eigenvalue amplitude was greater than one, the method was considered unstable.

Table I gives the parameters necessary to calculate the amplification matrix resulting from Monte Carlo calculations at low electric fields; Table II shows the parameters at $E=10 \mathrm{kV} / \mathrm{cm}$. Since the stability analysis assumes a uniform dc

TABLE II

Parameters Used to Calculate Amplification Matrix Entries at $E=10 \mathrm{kV} / \mathrm{cm}$

$$
\begin{aligned}
\tau_{n 1} & =1.45 \times 10^{-13} \mathrm{~s} \\
\tau_{n 2} & =5.6 \times 10^{-13} \mathrm{~s} \\
v_{1} & =1.65 \times 10^{7} \mathrm{~cm} / \mathrm{s} \\
v_{2} & =1.657 \times 10^{6} \mathrm{~cm} / \mathrm{s} \\
\tau_{e 2} & =1.74 \times 10^{-13} \mathrm{~s} \\
\tau_{e(2-1)} & =5.0 \times 10^{-13} \mathrm{~s} \\
\tau_{e(2-1)}^{*} & =9.2 \times 10^{-14} \mathrm{~s}
\end{aligned}
$$

$$
\begin{aligned}
\tau_{m 1} & =6.3 \times 10^{-14} \mathrm{~s} \\
\tau_{m 2} & =3.3 \times 10^{-14} \mathrm{~s} \\
\tau_{e 1} & =2.43 \times 10^{-12} \mathrm{~s} \\
\tau_{e(1-2)} & =7.78 \times 10^{-14} \mathrm{~s} \\
\tau_{e(1-2)}^{*} & =4.0 \times 10^{-13} \mathrm{~s} \\
w_{1} & =0.236 \mathrm{eV} \\
w_{2} & =0.0698 \mathrm{eV}
\end{aligned}
$$

Note. Asterisk denotes relaxation times with double bars in the energy equations. 
TABLE III

Results of Stability Analysis for GaAs at $T=500^{\circ} \mathrm{K}$

Using the Parameters of Tables I and II to Calculate the Amplification Matrix

\begin{tabular}{cccc}
\hline Method & $E(\mathrm{kV} / \mathrm{cm})$ & $D X(\mu \mathrm{m})$ & $\begin{array}{c}\text { Maximum } \\
\text { allowable } D T(\mathrm{~s})\end{array}$ \\
\hline FTUD & 0.0 & $0.01,0.02$ & Always unstable \\
FTUD & 10.0 & 0.01 & Always unstable \\
FTUD & 10.0 & 0.02 & $0.7 \times 10^{-14}$ \\
ML & 0.0 & 0.01 & $2.0 \times 10^{-14}$ \\
ML & 0.0 & 0.02 & $4.5 \times 10^{-14}$ \\
ML & 10.0 & 0.01 & $1.0 \times 10^{-14}$ \\
ML & 10.0 & 0.02 & $2.5 \times 10^{-14}$ \\
\hline
\end{tabular}

solution upon which the error terms are superimposed, it is expected that the analysis using the parameters in Table I more accurately reflects the stability restriction for IMPATT diode simulation since spatial uniformity of all the simulation variables occurs in the undepleted region where the electric field and per-carrier energies are low. At higher fields, significant spatial gradients exist and the stability restrictions in practice differ from those predicted by this analysis. Since for largesignal IMPATT simulation it has been found that instability most likely occurs in the low-field, low-energy limit, the analysis using Table I should give the approximate maximum allowable $\Delta t$ for a stable simulation provided the lowenergy limit is approched at some point during the RF cycle.

Table III presents the results of the stability analyses of the two numerical methods; here FTUD denoes forward time-upwind drift differencing and ML denotes the modified Lax numerical method. In the low-field limit it is found that the FTUD method is always unstable, no matter what time step is used. This is in accord with results previously obtained for the single-valley case [8], where it was found that this numerical method is unstable when applied to the energy and velocity transport equations. However, at $E=10 \mathrm{kV} / \mathrm{cm}$ the method is stable provided the space step is not too small. In practice, large-signal simulations using FTUD differencing are stable so long as the electric field does not become too low at any time in the cycle. However if the diode is driven with a larger ac voltage magnitude, a point is always reached where instability occurs.

On the other hand, Table III shows that the modified Lax method is stable even in the low-field limit. In practice, it has been found that the ML method is always stable provided a suitable time step is used; instability is only observed when the initial guess provided to the simulation is very poor.

The ML method provides stability by introducing numerical diffusion in the energy and velocity equations. The artificial diffusion coefficient is given by [6]:

$$
D=\frac{\Delta x^{2}}{2 \Delta t}\left[1-\frac{v^{2} \Delta t^{2}}{\Delta x^{2}}\right]
$$


Typically the second term in (15) is small so that

$$
D \approx \frac{\Delta x^{2}}{2 \Delta t}
$$

The Courant-Friedrich-Lewy stability condition requires that

$$
\Delta t \leqslant \frac{\Delta x}{v_{\mathrm{th}}}
$$

where $v_{\mathrm{th}}$ is the thermal velocity of carriers. Combining (16) and (17) yields:

$$
D \geqslant \frac{(\Delta x) v_{\text {th }}}{2} .
$$

It is clear from (18) that numerical diffusion is reduced by choosing a smaller space step and using the largest $\Delta t$ allowable for that $\Delta x$ value. To test this idea, simulations of a GaAs double-Read IMPATT diode $0.7 \mu \mathrm{m}$ long were carried out at $60 \mathrm{GHz}$, both using the FTUD and ML methods. In addition, simulations using the ML method were carried out using two different $\Delta x$ values. For this particular structure, the maximum efficiency point occurs before the electric field in the device collapses sufficiently to cause instability using the FTUD method so that the maximum efficiency points can be compared.

The large-signal simulations were carried out as follows. First, a de solution of the transport equations was obtained for a bias current density of $10 \mathrm{kA} / \mathrm{cm}^{2}$; this solution was the starting point for the large-signal program. A $60 \mathrm{GHz}$, sinusoidal RF voltage was impressed across the device, while maintaining the bias current at $10 \mathrm{kA} / \mathrm{cm}^{2}$. The simulation progressed until a periodic solution was obtained, i.e., the diode state at the beginning of an $\mathrm{RF}$ voltage cycle was the same as the state at the end of the cycle. The simulation variables and diode operating voltage $V_{\text {op }}$ adjusted to the values necessary to obtain a periodic solution. By Fourier analyzing the resulting waveforms, the RF power generated by the device and the conversion efficiency were calculated.

The choice of boundary conditions is critical for these simulations. For the cases presented here, Dirichlet boundary conditions were used for carrier concentrations at outflow points, and for per-carrier velocities and energies, at inflow points. The velocities at outflow points were set to maintain a Neumann boundary condition on the particle currents. The carrier concentrations at inflow points and the percarrier energies at outflow points were set by linear extrapolation, i.e., the values at the first two meshpoints adjacent to the boundary were linearly extrapolated back to the boundary point. These boundary conditions have been found suitable for double-drift IMPATT diode simulation; however, they typically must be modified to simulate other structures. The important consideration is to maintain a smooth transition to the boundary value for all the simulation variables. 


\section{TABLE IV}

Large-Signal Results at Maximum Efficiency Point for GaAs Double-Read Structure Simulated at $f=60 \mathrm{GH} 2, T=500^{\circ} \mathrm{K}$

\begin{tabular}{cccccc}
\hline Method & $D X(\mu m)$ & $D T(\mathrm{~s})$ & $V_{\text {RF }}(V)$ & $V_{\text {op }}(V)$ & $\begin{array}{c}\eta_{\max } \\
\text { (Percent) }\end{array}$ \\
\hline FTUD & 0.02 & $2.0 \times 10^{-14}$ & 12.5 & 21.7 & 16.29 \\
ML & 0.02 & $3.0 \times 10^{-14}$ & 12.5 & 22.4 & 12.93 \\
ML & 0.01 & $2.0 \times 10^{-14}$ & 12.5 & 22.8 & 16.69 \\
\hline
\end{tabular}

Table IV shows the large-signal results at the maximum efficiency points. It is seen that adequate agreement using the ML method is obtained only if the space step is reduced to $\Delta x=0.01 \mu \mathrm{m}$, thercby reducing the amount of numerical diffusion according to (18). Also shown are the time steps used for these simulations which are typical values used with each associated space step for IMPATT simulations. Of course when a smaller $\Delta x$ is used, the maximum allowable time step is also reduced according to (17). Since the FTUD method is known to have less numerical diffusion that the Lax method, the results obtained using the FTUD method were considered the standard by which the accuracy of the ML method could be judged.

The results of Table IV show that the ML method may be used, provided a sufficiently small space step $\Delta x$ is chosen and provided the maximum allowable time step $\Delta t$ is used for that particular space step. Other simulations of GaAs hybrid structures at 60 and $94 \mathrm{GHz}$ using both methods have also confirmed that numerical diffusion in the ML method is suitably small, provided a small enough space step is used.

\section{CONCLUSIONS}

It is desirable to use a one-step numerical method for energy-momentum computer simulation since the time step is restricted to the order of $10^{-14} \mathrm{~s}$. The forward time-upwind drift differencing method is useful provided the electric field does not become too low during the simulation; however, this method always becomes unstable in the low-field, low-energy limit. A modified Lax differencing method is presented which uses forward time-upwind drift differencing for the particle equation, but the Lax method for the velocity and energy equations. This method has the advantage that the particle equation is conservative. It is found that this method is stable in the low-field limit and that the numerical diffusion introduced by the Lax method may be reduced to acceptable levels by using a sufficiently small space step. 


\section{REFERENCES}

1. R. K. Mains, G. I. Haddad, ANd P. A. Blakey, IEEE Trans. Electron Devices, ED-30 (1983), 1327.

2. R. K. Mains, M. El-Gabaly, G. I. Haddad, and J. P. Sun, Comparison of theoretical experimental results for millimeter-wave GaAs IMPATTs, IEEE Trans. Electron Devices, ED-31 (1984), 1273.

3. M. El-Gabaly, R. K. Mains, and G. I. Haddad, Effects of doping profile on GaAs double-drift IMPATT diodes at 33 and $44 \mathrm{GHz}$ using the energy-momentum transport model," IEEE Trans. Microwave Theory and Techniques MTT-32 (1984), 1353.

4. M. A. El-Gabaly, R. K. Mains, and G. I. Haddad, Effect of doping profile variation on GaAs hybrid and double-read IMPATT diode performance at 60 and $94 \mathrm{GHz}$, IEEE Trans. Microwave Theory and Techniques MTT-32 (1984), 1342.

5. K. HuanG, "Statistical Mechanics," p. 95, Wiley, New York, 1963.

6. P. J. Roache, "Computational Fluid Dynamics," Hermosa, Albuquerque, N. M. 1976.

7. P. E. Bauhahn and G. I. Haddad, IEEE Trans. Electron Devices ED-24 (1977), 634.

8. R. K. Froelich, "Computer Modeling of Millimeter-Wave IMPATT Diodes," Ph.D. dissertation, University of Michigan, Ann Arbor, 1982.

9. D. PotTer, "Computational Physics," p. 50, Wiley, New York, 1977. 